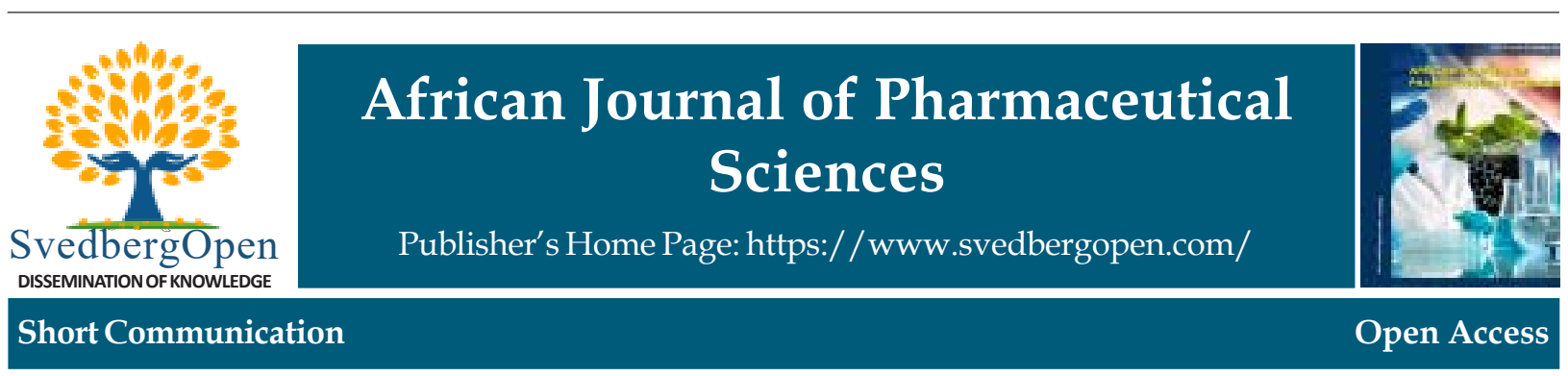

\title{
Target Therapy, from its Initial Clinical Breakthroughs to Current Hot Spot
}

\author{
George $\mathrm{Zhu}^{1^{*}}$ \\ ${ }^{1}$ Tehran University of Medical Sciences, Tehran, Iran. E-mail: sansan4240732@163.com
}

Article Info

Volume 1, Issue 1, September 2021

Received : 29 December 2020

Accepted : 15 June 2021

Published : 05 September 2021

doi: 10.51483/AFJPS.1.1.2021.11-15

\begin{abstract}
Epidermal growth factor (EGF) is a polypeptide hormone originally isolated from mouse submaxillary gland, which is a potent mitogen for a wide variety of culture cells of both epithelial and mesenchymal origin, and its important role in the proliferation, differentiation, and survival of neural and glial precursor cells. The physiological effects of EGF are mediated by an EGF receptor with tyrosine-specific protein kinase activity. The traditionally accepted view is that normal epidermal growth factor receptor (EGFR) is no tumorigenic, whereas mutated EGFR is oncogenic. Recently, it has been implicated that EGF could be beneficial for burn, wound healing, diabetic foot ulcer, and show an attractive perspective In addition, cosmetic containing EGF would be effective in improving the plasticity of skin, helps in anti-aging and whitening, and controls the amount of erythema and sebum in the skin. In our team we have successfully prepared a series of 206 bottles of Shampoo liquid and 20 bottles of recombinant human EGF (rhEGF) spray, and 4 bottles of EGFSilvadence ointment. The initial results indicated that prepared rhEGF is safe and available in clinical use. On the other hand, progress on the interaction of EGF with its mutated oncogenic receptors in the development of some cancers such as A431 human SCC cells, brain glioblastoma, breast, pancreas and lung cancers. In addition to a series of gefitinib, erlotinib, osimertinib and the CIMAvax-EGF vaccine, an antioncogenic receptor antibodybased fusion protein [for example Cetuximab-based IL-10 fusion protein, CmAb-(IL10)2] provide a potential strategy to improve cancer immunotherapy.
\end{abstract}

Keywords: EGF; EGFR and oncogenic receptor EGFR; Target therapy

(C) 2021 George Zhu. This is an open access article under the CC BY license (https://creativecommons.org/licenses/by/4.0/), which permits unrestricted use, distribution, and reproduction in any medium, provided you give appropriate credit to the original author(s) and the source, provide a link to the Creative Commons license, and indicate if changes were made.

$\mathrm{S}$ ince the discovery of oncogenic receptor by George Zhu from oncogenic PML/RARA fusion in the origin of a specific APL and androgen/oncogenic androgen receptor signaling in hormonal tumorigenesis in 1989s (Zhu, 1992; 2018; and 2019; Zhu et al., 2013;2016;2017; and 2020), many studies are focused on their clinical targeting therapy. At present, thousands of publications and over 100 global journals cited this scientific terminology "oncogenic receptor or oncogenic receptor (tyrosine) kinase". The traditionally accepted view is that normal Epidermal Growth Factor Receptor (EGFR) is no tumorigenic (Tang et al., 2000). Epidermal Growth Factor (EGF) consists of 53 amino acids residues and contain intramolecular disulfide bonds that are required for its biological activity (Cohen, 1962; Gregory, 1975; and Zhu et al., 2020). EGF can stimulate or inhibit proliferation and differentiation in a wide variety of cells, e.g., epidermal cells, corneal epithelial cells, fibroblasts, myofibroblasts, keratocytes and angiogenesis. EGF play a role in every tissue in the body during development and in the adult, the exact nature of this role is not clear. EGF interact with a specific EGF receptor which located at the cell surface (Stoscheck and King, 1986). In recent, it has been suggested that EGF could be beneficial for burn (Brown et al., 1989; and Wang et al., 1998), wound healing,diabetic foot ulcer (Afsbari et al., 2005; and Wong et al., 2018), and provide an attractive perspective. In addition,cosmetic containing EGF

\footnotetext{
* Corresponding author: George Zhu, Tehran University of Medical Sciences, Tehran, Iran. E-mail: sansan4240732@163.com
}

2789-5092/C 2021. George Zhu. This is an open access article distributed under the Creative Commons Attribution License, which permits unrestricted use, distribution, and reproduction in any medium, provided the original work is properly cited. 
could be effective to improve the plasticity, to remove wrinkle, to show whitening and anti-aging, and control of erythem amount and sebum amount on the human skin care. In this regard we (Zhu et al., 2020) have successfully prepared a series of 206 bottles of Shampo liquid (New Washing) and 20 bottles of recombinant human EGF(rhEGF) spray, and 4 bottles of EGF-Silvadence ointment into market. The initial results indicated that prepared rhEGF is safe and available in clinical wound healing and this may assist wound healing time.

On the other hand,tumor cells share oncogenic receptors (Robinson, 2008; and O'Connor, 2008). The oncogenic receptor EGFRvIII is like this case. EGFvIII is the deletion of exons 2-7 in the EGFmRNA and correspond to cDNA nucleotides 275-1075, which encode amino acids 6-276 in the EGFR protein. Deletion of 801 bp within the extracellular domain of the EGFR leads to in-frame trunction of the normal EGFR protein, resulting in a 145-kda receptor (Tang et al., 2000). Aggressive human glioma often express a truncated and oncogenic form of the epidermal growth factor receptor, known as oncogenic receptor EGFRvIII. Uptake of tumor cells derived microvesicles containing oncogenic EGFRvIII may alter the properties of endothelial cells and in relevance to tumor angiogenesis. Microvesicles containing the receptor could be released into cellular surrounding and blood of tumor-bearing mice, and can merge with the plasma membrane of cancer cells lacking EGFRvIII. This process leads to the transfer of oncogenic activity (Al-Nedawi et al., 2008). tEVs (tumor-derived extracellular vesicles) had potential to induce Cancer Stem Cells (CSCs) properties in normal tissue stem cells (Yan et al., 2014). EGFvIII-containing microvesicles could stimulate the proliferation of malignant glioma cells, stimulate activity of MAPK and AKT pathway, triggered production of VEGF and increased anchorageindependent growth of tumor cells, which was paralled by the increased phosphorylation of VEGFR2 (Montermini et al., 2015). In vitro 32D cells expressing high levels of EGFRvIII (32D/EGFRvIII) is capable of abrogating the IL-3dependent pathway in the absence of ligands. In vivo high levels of 32D/EGFRvIII formed large tumors in nude mice, whereas no tumors grew in mice injected with 32D/EGF or the parental 32D cells during a 2-month observation period (Tang et al., 2000). In mouse model,transgenic mice expressing EGFR L858R in type II pneunocytes developed atypical adenomatous hyperplasia and multifocal adenocarcinoma,and gefitinib inhibited tumorigenesis completely (Chihara et al., 2009). In our mice model, A subcutaneous nodule was observed in the application of continuous EGF injection, while no sign of nodule was shown following intramuscle EGF injection within 20 days (Zhu et al., 2020). All those data provide the evidence that EGFRvIII could play a pivotal role in oncogenesis.

A series of EGFR mutations in human lung adenocarcinoma has been described (Lynch et al., 2004; and Greulich et al., 2005). These mutations occur in four exons: substitutions for G719 in exon 18, in-frame deletions within exon 19, inframe insertions within exon 20, and substitutions for L858 in exon 21. Up to now, gefitinib (Iressa) and erlotinib (Tarceva) are the first generation EGFR Tyrosine Kinase Inhibitor (TKIs). Second generation EGFR TKI (afatinib and dacomitinib) were then developed more potent inhibitor. Moreover, gefitinib and erlotinib have a higher binding affinity for EGFR exon 19 deletion and exon 21(L858R) substitution mutation than for wild-type EGFR. Gefitinib and erlotinib inhibit the intracellular phosphorylation of EGFR tyrosine kinase, which blocks downstream signaling and EGF-dependent proliferation. Gefitinib and erlotinib were indicated for the treatments with metastatic Non-Small Cell Lung Cancer (NSCLC) harboring EGFR exon 19 deletions or exon 21 (L858R) substitution mutations. Drugs half-life were gefitinib 48 $\mathrm{h}$ and erlotinib $36.0 \mathrm{~h}$ respectively. Erlotinib is about $60 \%$ absorbed after oral administration and its bioavailability is significantly increased by food to almost 100\%. As a third-generation EGFR TKI, Osimertinib (Cross et al., 2014; and Finlay et al., 2014) binds to certain mutant forms of EGFR (T790M, L858R and exon 19 deletion) that predominate in NSCLC tumours who have progressed on or after first-line EGFR-TKI therapy. Therefore, approximately $10 \%$ of patients with NSCLC lung cancer patients containing activating EGFR mutation have been beneficial to dramatic clinically effective response to EGFR-TKIs. Konduri and colleagues (Konduri et al., 2016) reported five patients with metastatic lung cancer whose tumors harbored EGFR fusion,most commonly RAD5, are recurrent in lung cancer. Four of whom were treated with EGFR-TKI erlotinib with documented antitumor response for 5, 6, 8 and 20 months respectively. In our 2 patients with advanced lung cancers after oral gefitinib, we used gefitinib in keeping stable disease for $8+$ months in a female patient with lung adenocarcinoma (Zhu et al., 2017). In the phase III trial of 419 patients with advanced T790M positive NSCLC following osimertinib vs. platinum-based therapy, progression free survival in the osimertinib group was 8.5 months, compared to the platinum-based therapy group at 4.2 months (Mok et al., 2017).

The most promising, in Cuba, the CIMAvax-EGF vaccine trial is for people with stage IIIB or IV NSCLC who have been treated previously by one line of chemotherapy. CIMAvax-EGF is a therapeutic cancer vaccine composed of human recombinant EGF in yeast (hu-recEG) conjugated to the p64k Neisseria meningitides recombinant protein (recP64K) in Escherichia coli and Montanide ISAS1 as adjuvant (Rodriguez, 2016). At each immunization, patients received $2.4 \mathrm{mg}$ of hu-recEGF/recP64K/Montanide. Four doses was the minimum number of injections at $50 \%$ of the patients reaching 
the GAR status. Alternatively, patients who received at least four doses of CIMAvax-EGF had a significant effects on survival time. The vaccine induce antibodies against self EGFs that block EGF-EGFR interaction. In phase II clinical trial (Rodriguez et al., 2010), mean survival was 19.47 months in 20 patients with Good Antibody Responders (GAR), 4.97 months in Poor Antibody Responders (PARs) $(n=18)$, and 8.52 months in 37 controls. Long survival was found in all vaccinated patients (mean, 18.53 months) compared to randomized unvaccinated controls (mean, 7.55 months) in the group aged $<60$ years $(p<0.05)$.

A phase III clinical trial in patients with advanced III/IV NSCLC (Rodriguez et al., 2016), anti-EGF antibody titers was evaluated in 112 patients. 89 patients was GAR and 24 patients with super-good responders (titers > 1:64,000 sera dilution). Patients with GAR criterion had a significant survival benefits. Mean Survival Time (MST) was 10.83 months in the vaccine arm versus 8.86 months in the controls. CIMAvax-EGF is a very safe drug that could be a feasible intervention for long-term control of those NSCLC patients with tumors depending on the EGF, capable of produce a rapid and durable response. Like interleukin-2, recent finding that a Cetuximab-based IL-10 fusion protein (CmAb(IL10)2) was demonstrated to improve the effects of IL-10 to potentiate CD8+ T cell-mediated antitumor immunotherapy with reduced toxicity(Qiao et al., 2019). This is encouraging.

\section{Conclusion}

Thirty years ago, Zhu is the first to conduct that targeting therapy is mainly shift toward oncogenic receptors (also molecular "missile therapy" at that period) (Zhu G, 1992). Actually, at more earlier 1984, effects of monoclonal antibody 1083-17-1A of the IgG2a immunotherapy was initially trials on patients with metastatic gastrointestinal adenocarcinoma, 3 of 20 patients had no detectable disease for 10, 13 and 22 months. In 1994, mAb17 -1A (later named edrecolomab) was the first to show its clinical efficacy in increasing disease-free survival in resected colorectal cancer patients. Later advances, several anti-EpCAM antibodies have been developed (Catumaxomab, NEA125, ING101, adecatumumab and other EpCAM-specific immunotoxin). In 2009, the European Medicines Agency approved catumaxomab, which binds to oncogenic receptor EpCAM and enhances the immunological response against EpCAM-positive cells in malignant ascites. In addition, mutations in EpCAM could lead to decreased expression of EpCAM, loss of cell surface EpCAM, resulting in congenital tufting enteropathy. These results suggest that EpCAM is not an oncogene. Since then, more effective anti-EpCAM antibodies engineering are entered into clinical use.

Gleevec (imatinib), a breakthrough therapeutic orally tyrosine kinase inhibitor that targeting bcr-abl oncogene in chronic myeloid leukemia. Imatinib is also used for indication of GIST patients with oncogenic receptor tyrosine kinase PDGFR mutant/or HES and myeloproliferative neoplasm with oncogenic PDGFR fusion, and oncogenic receptor KIT (also stem cell factor receptor), and inhibits activation of PI3K, MAPK or PI3k/Akt pathway. In addition to target therapy directed to oncogenic receptor EGFRvIII, another burgeoning class of targeted chemotherapies called antibodydrug conjugates. Trastuzumab (Herceptin) and ado- trastuzumab emtansine (or Trastuzumab deruxtecan) enables lysosomal degradation of its cognate oncogenic receptor HER2 or release of prodrugs in HER2+ metastatic breast cancer via antibody-dependent cellular cytotoxicity (ADCC). The patients showed a significant survival benefits. More data, ONC201 is the first clinical bitopic antagonist of DRD2, an oncogenic receptor in brain and neuroendocrine tumors. Lenvatinib mesilate (lenvatinib) inhibits the multiple RTKs that target oncogenic receptor VEGFR1, 2, and other proto-oncogenic receptor tyrosine kinases, including PDGFa, Kit and RET inhibition, also shown antitumor activity against multiple tumor types, such as preclinical human thyroid cancer models. A bi-specific antibody (GSK3178022) to LRP6, a key oncogenic receptor, is capable of blocking stimulation of Wnt signaling in vitro, delays tumor growth in patient-derived RSPO fusion model of colorectal cancer. Targeting IGF-1R pro-oncogenic RTK signaling is still uncertain results. Thus, down regulating oncogenic receptors may be useful paradigm and perspective in currently the first or third-line setting of clinical standard therapy in those metastatic and advanced cancers.

\section{References}

Afsbari, M., Larijani, B., Fadayee, M. et al. (2005). Efficacy of Topical Epidermal Growth Factor in Healing Diabetic Foot Ulcer. Therapy, 2(5), 759-765.

Al-Nedawi, K.,Meehan, B., Micallef, J. et al. (2008). Intracellular Transfer of the Oncogenic Receptor EGFRvIII by Microvesicles Derived from Tumour Cells. Nat Cell Biol, 10, 619-624.

Brown, G.L.,Nanney, L.B.,Groffen, J. et al. (1989). Enhancement of Wound Healing by Topical Treatment With Epidermal Growth Factor. N Engl J Med, 321, 76-79. 
Chihara, E.,Takeda, H., Kubo, T. et al. (2009). Chemo Preventive Effect of Gefitinib on Non-smoking-related Lung Tumorigenesis in Activating Epidermal Growth Factor Receptor Transgenic Mice. Cancer Res, 69, 7088-7095.

Cohen, S. (1962). Isolation of a Mouse Submaxillary Gland Protein Accelerating Incisor Eruption and Eyelid Opening in the New-born Animal. J Biol Chem, 237, 1555-1562.

Cross, D.A., Ashton, S.E.,Ghiorghiu, S. et al. (2014). AZD9291, An Irreversible EGFR TKI, Overcomes T790M-mediated Resistance to EGFR Inhibitors in Lung Cancer. Cancer Discov, 4(9), 1046-61.

Finlay, M.R., Anderton, M., Ashton, S. et al. (2014). Discovery of a Potent and Selective EGFR Inhibitor (AZD9291) of both Sensitizing and T790M Resistance Mutations that Spares the Wild Type Form of the Receptor. J Med Chem, 57(20), 8249-67.

Gregory, H. (1975). Isolation and Structure of Urogastrone and its Relationship in Epidermal Growth Factor. Nature, 257, $325-327$.

Greulich, H.,Chen, T.H., Janne, P.A. et al. (2005). Oncogenic Transformation by Inhibitor-sensitive and -Resistant EGFR Mutants. PloS Med, 2(11), e313.

Konduri, K.,Gallant, J.N., Chae, Y.K. et al. (2016). EGFR Fusions as Novel Therapeutic Targets in Lung Cancer. Cancer Discov, 6, 601-611.

Lynch, T.J., Bell, D.W., Sordella, R. et al. (2004). Activating Mutations in the Epidermal Growth Factor Receptor Underlying Responsiveness of Non-small-cell Lung Cancer to Gefitinib. N Engl J Med, 350(21), 2129-39.

Mok, T.S.,Wu, Y.L., Ahn, M.J. et al. (2017). Osimertinib or Platinum-pemetrexed in EGFR T790M- positive Lung Cancer. NEngl J Med, 376, 629-640.

Montermini, L., Meehan, B., Garnier, D. et al. (2015). Inhibition of Oncogenic Epidermal Growth Factor Receptor Kinase Triggers Release of Exosome-like Exttacellular Vesicle and Impacts Their Phosphoprotein and DNA Content. J Biol Chem, 290(40), 24534-24546.

O’Connor, R. (2008). Concealed Cargo Within The Tumor Microenvironment: Microvesicles Disseminate Oncogenic Receptors Among Cancer Cells. Cancer Biol Ther, 7, 1350-51.

Qiao, J., Liu, Z.D., Fu, Y.X. et al. (2019). Targeting Tumors with IL-10 Prevents Dendritic Cell-Mediated CD8+ T Cell Apoptosis. Cancer Cell, 35, 901-915.

Robinson, B. (2008). Tumor Cells Share Oncogenic Receptors. J Cell Biol, 181, 570.

Rodriguez, P.C., Popa, X., Martinez, O. et al. (2016). A Phase III Clinical Trial of the Epidermal Growth Factor Vaccine CIMavax-EGF as Switch Maintenance Therapy in Advanced Non-small-cell Lung Cancer Patients. Clin Cancer Res, 22(15), 3782-3790.

Rodriguez, P.C., Rodrigue, G., Gonzalez, G. et al. (2010). Clinical Development and Perspectives of CIMAvax EGF, Cuban Vaccine for Non-small-cell Lung Cancer Therapy. MEDICC Rev, 12(1), 17-23.

Stoscheck, C.M., and King, L.E. (1986). Role of Epidermal Growth Factor in Carcinogenesis. Cancer Res, 46(3),1030-7.

Tang, C.K.,Gang, X.Q.,Wong, A.J. et al. (2000). Epidermal Growth Factor Receptor vIII Enhances Tumorigenicity in Human Breast Cancer. Cancer Res, 60(11), 3081-3087.

Wang, S.L., Guo, Z.R., Fu X.B. et al. (1998). Effects of Recombinant Human Epidermal Growth Factor on Healing of Chronic Ulcer Wound. Chinese J Traumat (in chinese), 14(6), 348-349.

Wong, W.K.R., Ng, K.L., Hu, X.H. et al. (2018). Authentic Human Epidermal Growth Factor: A Panacea for Wound Healing. EC Endocr Metab Res, 3(4), 138-146.

Yan, T., Mizutani, A.,Chen, L. et al. (2014). Characterization of Cancer Stem-like Cells Derived from Mouse Induced Pluripotent Stem Cells Transformed by Tumor-derived Extra-cellular Vesicle. J Cancer, 5(7), 572-584.

Zhu, G. (1992). Oncogenic Receptor Hypothesis (1989-91). VOA(Voice of America), 12, 31.

Zhu, G. (2018). Treatment of Patients with Advanced Cancer Following Chemotherapy and Traditional Medicine- Long Term Follow up of 75 Cases. Univ J Pharm Res, 3(3), 10-18. 
Zhu, G. (2018). EpCAM-An Old Cancer Antigen, Turned Oncogenic Receptor and Its Targeting Immunotherapy. Univ J Pharm Res, 3(2), 41-46.

Zhu, G. (2019). Vitamin A and its Derivatives-retinoic Acid and Retinoid Pharmacology. Am J Biomed Sci \& Res, 3(2), 162177.

Zhu, G., Mische, S.E., and Seigneres, B. (2013). Novel Treatment of Acute Promyelocytic Leukemia: $\mathrm{As}_{2} \mathrm{O}_{3}$, Retinoic Acid and Retinoid Pharmacology. Curr Pharm Biotechnol, 14(14), 849-858.

Zhu, G., Saboor-Yaraghi, A.A.,Yarden, Y. et al. (2016). Downregulating Oncogenic Receptor: From Bench to Clinic. Hematol Med Oncol, 1(1), 30-40.

Zhu, G., Musumecci, F., Byrne, P. et al. (2017). A Pilot Study of Lung Cancer Following Chemotherapy and Traditional Medicine: Report of 12 Cases. Lungs and Breath J, 1(3), 1-4.

Zhu, G., Musumeci, F., and Byrne, P. (2013). Induction of Thyroid Neoplasm Following Plant Medicine Marine Algae (Sargassum): A Rare Case and Literature. Curr Pharm Biotechnol, 14(9), 859-863.

Zhu, G., Xu, H.L., Zhi, Q.W. et al. (2020). Enhancement of Wound Healing by Topical Application of Epidermal Growth Factor in Animal Model. Univ J Pharm Res, 5(1), 12-20.

Cite this article as: George Zhu (2021). Target Therapy, from its Initial Clinical Breakthroughs to Current Hot Spot. African Journal of Pharmaceutical Sciences, 1(1), 11-15. doi: 10.51483/AFJPS.1.1.2021.11-15. 